\title{
In-vitro Studies of Pentoxifylline Controlled-Release from Hydrophilic Matrices
}

\author{
B. M. Rahman ${ }^{\dagger}$, M. A. Islam, M. I. I. Wahed, Maruf Ahmed, \\ R. Islam, R. K. Barman, A. S. M. Anisuzzaman, and Proma Khondkar \\ Department of Pharmacy, Rajshahi University, Rajshahi-6205, Bangladesh \\ Received 31 August 2008, accepted in final revised form 15 February 2009
}

\begin{abstract}
Methocel K15 MCR (a modified hydroxypropylmethylcellulose) matrix tablets of pentoxifylline using microcrystalline cellulose (MCC), starch and lactose were prepared by wet granulation process. There was no significant difference in drug release between the hydrophilic matrices when the Methocel K15 MCR concentration was modified in low percentage. The release of pentoxifylline was influenced by the presence of microcrystalline cellulose, and by the different concentrations of starch and lactose. The data obtained proved that the formulation with 12.5\% Methocel K15 MCR and 20\% lactose is more useful for a controlled release pentoxifylline, due to the percentage released after 8 hours is nearly to $65 \%$. There was no significant change on release profile after three months storage of tablets. The best-fit release kinetics was achieved with the Higuchi model, followed by the first-order plot.
\end{abstract}

Keywords: Pentoxifylline; Controlled release; Methocel K15 MCR.

(C) 2009 JSR Publications. ISSN: 2070-0237 (Print); 2070-0245 (Online). All rights reserved.

DOI: $10.3329 /$ jsr.v1i2.1080

\section{Introduction}

Hydrophilic matrices containing swellable polymers are referred to as hydrogel matrices, swellable controlled-release systems or hydrophilic matrix tablets. A number of polymers have been investigated to develop in situ gel-forming systems, due to the ability of these hydrogels to release an entrapped drug in aqueous medium and to regulate the release of such drug by control of swelling and cross-linking [1-3].

These systems draw attention in the search for improved patient compliance and decreased incidence of adverse drug reactions. Under ideal conditions, a controlledrelease formulation maintains therapeutic blood level of a drug for a specific period of time. Oral controlled-release dosage forms have been developed and studied to restrict these systems to specific regions of the gastrointestinal tract as well as to improve the pharmacological activity and to reduce toxic effects [4]. One method of fabricating

\footnotetext{
${ }^{\dagger}$ Corresponding author: bmokaddes@yahoo.com
} 
controlled-release formulations is by the incorporation of the drug in a matrix containing a hydrophilic, rate-controlling polymer [5, 6]. Hydroxypropylmethylcellulose (HPMC) is the polymer most widely used as the gel-forming agent in the formulation of solid, liquid, semisolid and even controlled-release dosage forms. Water penetration, polymer swelling, drug dissolution, drug diffusion and matrix erosion from these dosage forms are controlled by the hydration of HPMC, which forms a gel barrier through which the drug diffuses $[7,8]$. The adjustment of the polymer concentration, the viscosity grade and the addition of different types and levels of excipients to the HPMC matrix can modify the drug release rate. The importance of the diffusion layer for a swollen HPMC matrix was illustrated in a mathematical model [9]. In addition, the influence of technological variables on drug release from HPMC matrices was reviewed [10]. The influence of high viscosity grade of HPMC on the drug release from matrix tablets was reported by [11].

Pentoxifylline is a tri-substituted xanthine derivative designated chemically as 1-(5oxohexyl)-3,7-dimethylxanthine that, unlike theophylline, is a hemorrheologic agent, i.e. an agent that affects blood viscosity. Pentoxifylline is soluble in water and ethanol, and sparingly soluble in toluene. Pentoxifylline causes hemodynamic improvement by increasing erythrocyte flexibility and decreasing blood viscosity, thereby increasing the oxygen supply to muscles [12]. The precise mode of action of Pentoxifylline and the sequence of events leading to clinical improvement are still to be defined.

The release of pentoxifylline from matrix tablets containing palmitic or behenic acids, as waxes, and prepared via cogrinding was investigated and the release rate decreased with an increased grinding time and increased significantly with an increased proportion of the drug [13]. Despite the influence of some technological variables have been already described, one goal of this study was to develop uncoated Methocel K15 MCR matrix tablets by the wet granulation process, evaluating the relationship and effect of coexcipients. Microcrystalline cellulose and starch were chosen as release modifiers and lactose as water-soluble diluent.

\section{Materials and Methods}

\subsection{Materials}

Pentoxifylline was purchased from Zentiva International, a, s, Slovakia. Hydroxypropyl methylcellulose (Methocel K15 MCR) was supplied by Colorcon, India. Microcrystalline cellulose (Avicel pH 101) was obtained from Angel Associate, Taiwan. Lactose was purchased from Fonterra Ltd. Newzeland. Magnesium stearate was purchased from Peter Greven, c.v. Netherlands. All other reagents were analytical or pharmaceutical grade.

\subsection{Preparation and characterization of matrix tablets}

The detailed compositions of Methocel K15 MCR matrix tablet formulations are given in Table 1. Methocel K15 MCR, at different ratios was blended with the pentoxifylline, 
microcrystalline cellulose (MCC), starch and/or lactose, in a planetary mixer for 5 minutes. There after, the powders were granulated with $40 \% \mathrm{w} / \mathrm{v}$ purified water, sieved using a 16 mesh screen, and the granules obtained dried in a hot air oven at $60^{\circ} \mathrm{C}$ for 2 hours. Finally, the granules were dried and sieved using 20-mesh screen before tableting. Tablets of approximately $600 \mathrm{mg}$ weight each were prepared from these granules after addition of magnesium stearate (0.5\%). Tablets were compressed using a single punchtableting machine (Korsch-Berlin, EK/0, Frankfort, Germany) equipped with $13 \mathrm{~mm}$ shallow round punches. Three batches were prepared for each formulation. The physical properties of the matrix tablets are given in Table 2. The weight variation of the tablets was evaluated on 20 tablets using an electronic balance (Mettler Toledo, Switzerland). Friability was determined using $6 \mathrm{~g}$ of tablets in a friabilator (Pharmatest, Germany) for 4 minute at a speed of $25 \mathrm{rpm}$. For each formulation the hardness of 10 tablets was also evaluated using a hardness tester (Erweka, Germany). The tablet hardness ranged from 8 to $11 \mathrm{~kg}$. The thickness of the tablets was measured on 10 tablets with a Vernier Calipter (Mitutoyo, Japan).

Table 1. Composition (in mg) of 400-mg Pentoxifylline matrix tablet.

\begin{tabular}{cllll}
\hline $\begin{array}{c}\text { Formulation of } \\
\text { Pentoxifylline Tablet } \\
\text { ( PT) }\end{array}$ & $\begin{array}{c}\text { Methocel K15 } \\
\text { MCR }\end{array}$ & Lactose & MCC & Starch \\
\hline PT 1 & 75 & 120 & - & \\
PT 2 & 71.25 & 120 & 3.75 & - \\
PT 3 & 69.37 & 120 & 5.63 & - \\
PT 4 & 75 & - & - & 120 \\
PT 5 & 71.25 & - & 3.75 & 120 \\
PT 6 & 69.37 & - & 5.63 & 120 \\
PT 7 & 75 & 60 & - & 60 \\
PT 8 & 71.25 & 60 & 3.75 & 60 \\
PT 9 & 69.37 & 60 & 5.63 & 60 \\
\hline
\end{tabular}

\subsection{In-vitro drug dissolution studies}

Drug release profiles were evaluated in vitro using a dissolution test apparatus (Erweka, Germany). The USP paddle method was selected to perform the dissolution profiles of pentoxifylline from Methocel K15 MCR. The same test for all the formulations was carried out in $1000 \mathrm{~mL}$ water, maintained at $37 \pm 0.5^{\circ} \mathrm{C}$ at a paddle rotation speed of 100 rpm. The $10 \mathrm{~mL}$ of release medium was taken as a sample at preselected time intervals up to 8 hours monitored progress of the dissolution and replaced by a fresh $10 \mathrm{ml}$ of medium. The filtered sample solutions were analyzed for pentoxifylline by UV absorbance at 274 nm using a UV-Visible Spectrophotometer. Cumulative percentage of drug release was calculated and the mean of three determinations was used in data analysis. 


\subsection{Release kinetics}

To study the mechanism of drug release from the matrix tablets, the release date were fitted to the following equations:

Zero-order equation: $Q=Q_{0^{-}} k_{0} t$

where $Q$ is the amount of drug release at time $t$, and $k_{0}$ is the release rate;

First-order equation: $\ln Q=\ln Q_{0}-k_{1} t$

where $k_{1}$ is the release rate constant;

Higuchi's equation: $Q=k_{2} t^{1 / 2}$

where $Q$ is the amount of drug release at time $t$, and $k_{2}$ is the diffusion rate constant.

\subsection{Stability study}

A stability test was conducted by storing tablets in Alu-Alu strip at $30^{\circ} \mathrm{C} \pm 60 \% \mathrm{RH}$ and $40^{\circ} \mathrm{C} \pm 75 \% \mathrm{RH}$. The content of pentoxifylline and the dissolution of drug from these matrix tablets were tested after three months from storing. The assay of pentoxifylline and the dissolution study followed the same procedure as previously described.

\section{Results and Discussion}

\subsection{Physical characterization of the matrix tablets}

Actual drug content, friability, thickness, hardness and weight of the formulated tablets are described in Table 2. The weight deviation of the nine matrix tablets was acceptable due to the granule flowability properties. The flow characteristics were found satisfactory. The minimal friability obtained confirmed the suitability of the wet-granulation technology for the preparation of these HPMC matrices.

Table 2. Physical properties of pentoxifylline matrix tablets.

\begin{tabular}{llllll}
\hline Tablets & $\begin{array}{c}\text { Mean wt. } \\
(\mathrm{mg})\end{array}$ & $\begin{array}{c}\text { Actual drug } \\
\text { content }(\%)\end{array}$ & $\begin{array}{c}\text { Mean } \\
\text { thickness } \\
(\mathrm{mm})\end{array}$ & $\begin{array}{c}\text { Friability } \\
(\% \text { loss })\end{array}$ & $\begin{array}{c}\text { Mean } \\
\text { hardness } \\
(\mathrm{kg})\end{array}$ \\
\hline PT 1 & $604.8 \pm 0.021$ & $102.16 \pm 0.21$ & $4.75 \pm 0.17$ & 0.04 & $8.2 \pm 0.22$ \\
PT 2 & $606.5 \pm 0.004$ & $100.45 \pm 0.40$ & $4.71 \pm 0.15$ & 0.05 & $9.5 \pm 0.20$ \\
PT 3 & $601.7 \pm 0.031$ & $97.98 \pm 0.34$ & $4.65 \pm 0.18$ & 0.11 & $8.8 \pm 0.41$ \\
PT 4 & $608.8 \pm 0.045$ & $103.14 \pm 0.09$ & $4.72 \pm 0.11$ & 0.02 & $9.9 \pm 0.27$ \\
PT 5 & $603.6 \pm 0.035$ & $98.38 \pm 0.06$ & $4.67 \pm 0.12$ & 0.09 & $10.5 \pm 0.42$ \\
PT 6 & $608.8 \pm 0.028$ & $101.44 \pm 0.18$ & $4.76 \pm 0.09$ & 0.12 & $9.7 \pm 0.34$ \\
PT 7 & $596.2 \pm 0.151$ & $99.67 \pm 0.27$ & $4.68 \pm 0.04$ & 0.04 & $10.7 \pm 0.11$ \\
PT 8 & $611.4 \pm 0.037$ & $98.75 \pm 0.29$ & $4.65 \pm 0.08$ & 0.07 & $9.4 \pm 0.29$ \\
PT 9 & $598.8 \pm 0.019$ & $101.57 \pm 0.20$ & $4.73 \pm 0.06$ & 0.02 & $10.2 \pm 0.55$ \\
\hline
\end{tabular}




\subsection{Effect of lactose}

Lactose is the most useful filler used for tablet formulations. It is water-soluble and would modify the drug release for undergoing dissolution. Drug release from the tablets compressed with pentoxifylline, Methocel K15 MCR, lactose and MCC, are shown in Fig. 1.

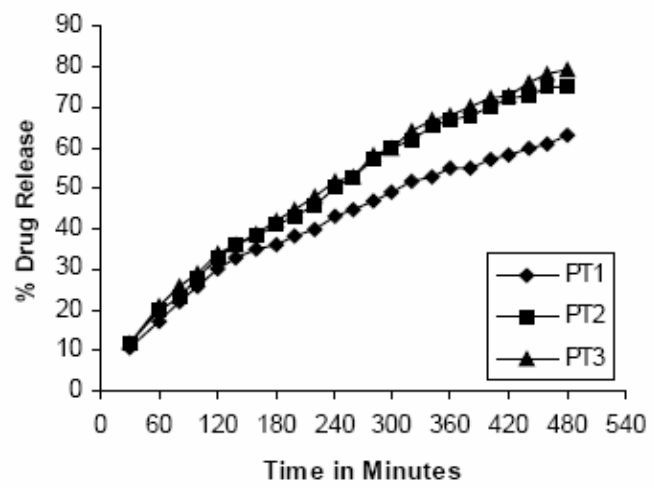

Fig. 1. Percent of pentoxifylline release rate from HPMC matrix tablets, containing $20 \%$ of lactose.

While the batch PT1 (0\% MCC-20\% lactose), the 63\% drug was released after $8 \mathrm{~h}$, when MCC was incorporated in the formulations PT2 (5\% MCC-20\% lactose), and PT3 (7.5\% MCC-20\% lactose), the release rate was markedly increased up to $75 \%$ and $79 \%$ respectively, compared to a PT1 tablet without MCC. In this case, the swelling behavior of MCC allowed further penetration of the aqueous medium, resulting in rapid erosion of the polymer matrices. In addition, lactose in aqueous solution plays a role as important physical barrier, affecting the release kinetics, by reducing the tortuosity of the diffusion pattern of the drug [14]. However, if it were mixed with Methocel K15 MCR, the polymer concentration would lead the release rate of the drug. Thus, it was expected that decreasing the Methocel K15 MCR concentration, being constant the lactose concentration could increase the release rate of Pentoxifylline, as shown in Fig. 1.

\subsection{Effect of starch}

Starch is water swellable by nature. The presence of starch in a Methocel K15 MCR matrix tablet could modify the release rate, due to the disintegration phenomenon is based on the fast water uptake, followed by the Methocel K15 MCR swelling [15]. Thus, the comparative influence of the co-excipients starch and MCC as release modifiers was evaluated. Fig. 2 shows plot of the cumulative amount of drug released against time for the formulations PT4, PT5 and PT6.

It was observed that the different concentrations of Methocel K15 MCR on the drug release rate were not significant, due to the similar behavior of the three formulations 
under the same dissolution conditions. In this case, small variations on the Methocel K15 MCR concentration would not affect the profile release of the model drug, despite of another study where was reported that the increase of HPMC content with the same drug concentration resulted in a decreased release rate of drugs [16]. The results obtained

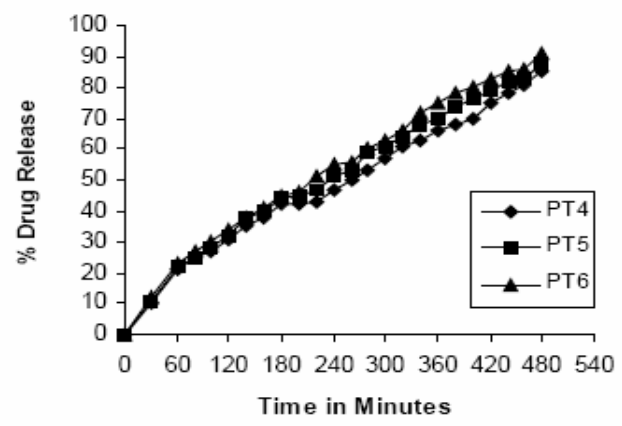

Fig. 2. Percent of pentoxifylline release rate from HPMC matrix tablets, containing $20 \%$ of starch.

herein show clearly that the drug released at 8 h of the batch PT4 (0\% MCC-20\% starch) was slightly higher, compared with the batches PT5 (5\% MCC-20\% starch) and PT6 (7,5\% MCC-20\% starch), indicating that MCC did not produce any relevant effect on the drug release rate. We assume, in this particular case, that MCC works as diluent creating the desired bulk as well as giving the right compression characteristics [17]. Thus, release rate of pentoxifylline from PT4, PT5, and PT6 would be mainly controlled by the hydration and swelling properties of Methocel K15 MCR and starch, which forms a gel layer that controls the water penetration and drug diffusion.

\subsection{Effect of lactose: starch mixture}

The last three batches PT7, PT8 and PT9 were formulated by mixing lactose and starch in a 1:1 ratio, having in mind that each excipient by itself modified the release rate of the drug, in agreement with the results described in Figs. 1 and 2. Fig. 3 depicts the release profile of PT7 (0\% MCC-10\% starch-10\% lactose), MT8 (5\% MCC-10\% starch-10\% lactose), and MT9 (7.5\% MCC-10\% starch-10\%lactose).

The percentage of drug released at $8 \mathrm{hr}$ from PT8 and PT9 matrices was more than $85 \%$ and $90 \%$, while the percentage drug release from PT7 matrix was less than $72 \%$; clearly showing that in this particular case the presence of MCC plays an important role as filler as well as a release modifier $[18,19]$. These tablets containing a $50 \%$ less of starch and a $50 \%$ less of lactose showed a higher release rate of pentoxifylline compare with the matrix tablets formulated only with starch (PT4-PT6) or lactose (PT1-PT3). By this observation, we can conclude that lowering the amount of starch; it was enough to produce the dissolution process on a very good controlled-release rate. Moreover, decreasing the amount of lactose, did not affect the path diffusion of the drug, due to the good release kinetic obtained. 


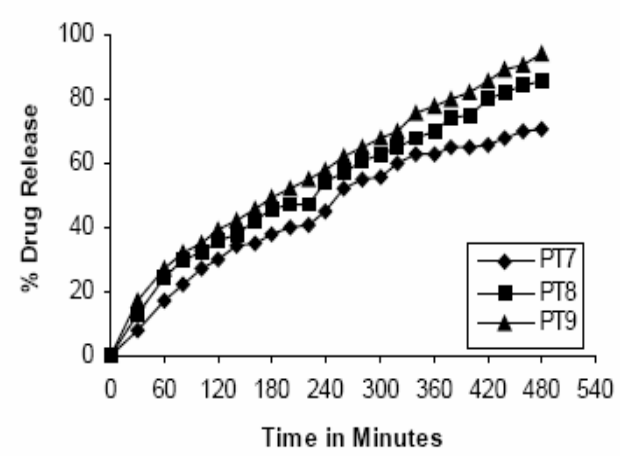

Fig. 3. Percent of pentoxifylline release rate from HPMC matrix tablets, containing $10 \%$ of starch and $10 \%$ of lactose.

\subsection{Drug release kinetics}

When percent release was plotted against time (Fig. 4), no straight lines were obtained indicating that pentoxifylline release did not follow the zero order kinetics. First order plot (log \% remaining vs. time) showed a linear released pattern (Fig. 5). In contrast, when percent release was plotted against square root of time (Fig. 6), release profile showed linear relationship. This confirms that matrix tablets were released in a Higuchian diffusion fashion.

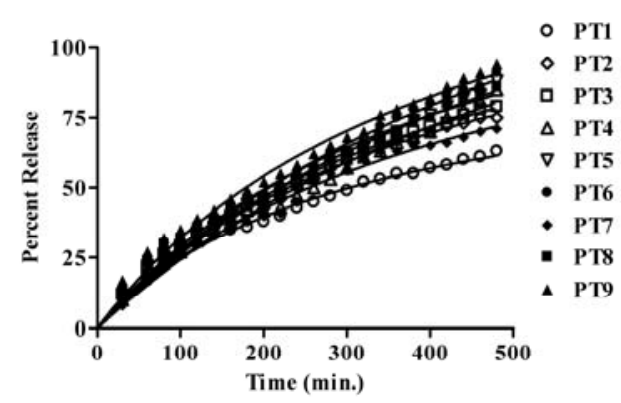

Fig. 4. Zero order release profile of pentoxifylline from polymeric matrix tablets.

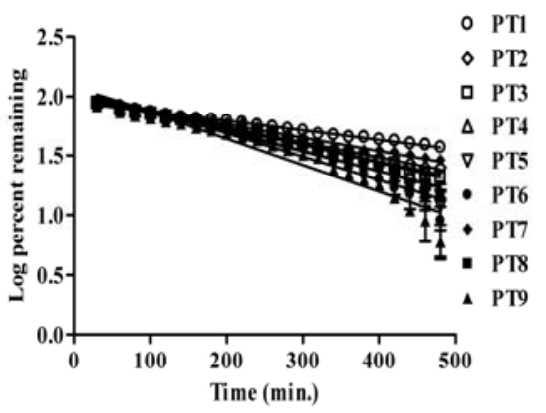

Fig. 5. First order release profile of pentoxifylline from polymeric matrix tablets.

The calculated correlation coefficient $\left(r^{2}\right)$ of the percent drug release versus square root of time was between 0.9811-0.9969 whereas those of the percent drug release versus time and log percent drug remained versus time were between 0.9627-0.9932 and 0.92780.9960 , respectively. This implied that the best-fit release of drug from the prepared tablets accordingly followed Higuchi model. The percent release versus time plot suggests 
that formulation (PT1) with 12.5\% Methocel K15 MCR and 20\% lactose is being preferred proportion in the preparation of pentoxifylline matrix tablets for the therapy, due to the percentage released after 8 hours is nearly to 65\% [20]. Dissolution data fit a firstorder kinetics, despite of quite high correlation coefficients were obtained with Higuchi kinetic model.

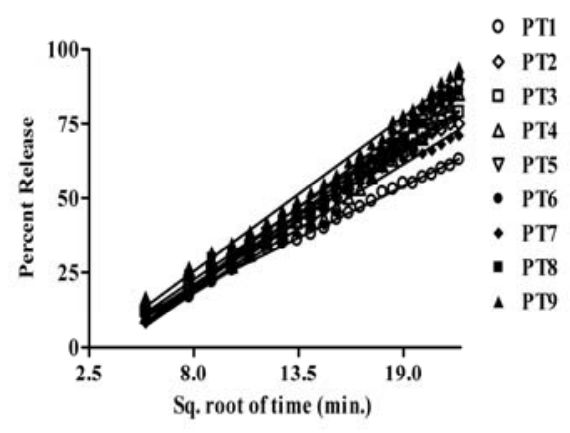

Fig. 6. Higuchi release profile of pentoxifylline from polymeric matrix tablets.

\subsection{Lot reproducibility and stability test}

Three batches of each formulation were prepared and the dissolution rate of pentoxifylline was evaluated under the same conditions. The resulting release profiles of pentoxifylline from these three different batches of each matrix formulation (PT1-PT9) showed no significant difference among the release profiles for each set of three batches, indicating that this manufacturing process is reliable and reproducible.

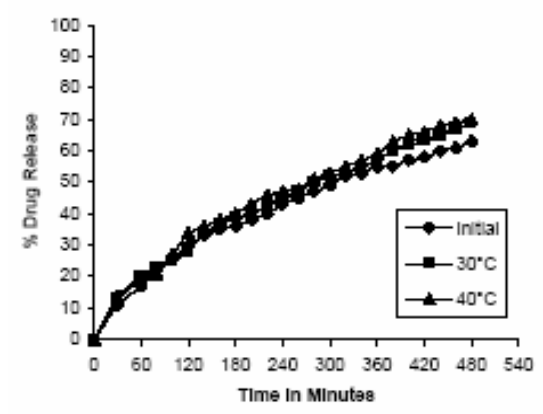

Fig. 7. Percent of pentoxifylline release rate from HPMC matrix tablets, containing $20 \%$ of lactose stored at $30^{\circ}$ $\mathrm{C}$ and $40^{\circ} \mathrm{C}$ after 3 months.

The stability of pentoxifylline in these matrix tablets was evaluated over three months at two different temperatures. There was no physical change in the tablets, moreover, there was insignificant pentoxifylline degradation in the nine formulated tablets, suggesting that the drug is stable in the Methocel K15 MCR tablets (Fig. 7). Apparently, the drug release from these matrices does not change after storage for this period of time. 


\section{Conclusions}

Pentoxifylline release matrices were prepared successfully utilizing Methocel K15 MCR as a carrier. From the technological point of view, the wet granulation method enables the preparation of these matrices. The physical properties described in Table 2 were found to be optimal for the manufacturing process. There was no significant difference in drug release between the hydrophilic matrices when the Methocel K15 MCR concentration was modified in low percentage. Drug release from swollen matrices was principally regulated by starch (20\%) or lactose (20\%), even on the presence of MCC at different levels (5\% or 7.5\%). However, when starch (10\%) and lactose (10\%) were mixed at lower concentration in a ratio $1: 1$, MCC (5\% or $7.5 \%)$ appeared to control the drug release from the matrices. Clearly, each of these components was capable of interacting to some extent with each other to control drug release. The best-fit release kinetics with the highest correlation coefficients was achieved with the Higuchi plot followed by the first-order equations, over 8 h. The data described in Figs. 1, 2, and 3 proved that the formulation, PT1, is more useful for a controlled release of pentoxifylline, due to the percentage released after $8 \mathrm{~h}$ is nearly to $65 \%$. The formulations with their release profile were not affected when subjected to different stability conditions after three months storage of tablets. The results obtained herein were in USP 28 describing the release rates of pentoxifylline controlledrelease tablets.

\section{Acknowledgments}

The authors are grateful to Product Development and Validation Department, Square Pharmaceuticals Ltd., Bangladesh for the financial support and facilities to carry out this study.

\section{References}

1. L. Krowczynsky, Extended-Release Dosage Form (CRC Press, Boca Raton, FL, 1987).

2. Y. W. Chien, Novel Drug Delivery Systems, 2nd Edition (Marcel Dekker, New York, 1992).

3. M. N. V. Ravi Kumar and N. Kumar, Drug Dev. Ind. Pharm. 27, 1 (2001). doi:10.1081/DDC-100000124

4. T. J. Roseman and N. F. Cardinelli, Controlled-release Technologies, Vol. 1 (A. F. Kydonieus, ed), (CRC Press, Boca Raton, FL, 1980).

5. F. Veiga, T. Salsa, and E. Pina, Drug Dev. Ind. Pharm. 24, 1 (1988). doi:10.3109/03639049809082346

6. P. Colombo, Adv. Drug Del. Rev. 11, 37 (1993). doi:10.1016/0169-409X(93)90026-Z

7. K. C. Sung, P. R. Nixon, J. W. Skoug, T. R. Ju, P. Gao, E. M. Topp, and M. V. Patel, Int. J. Pharm. 142, 53 (1996). doi:10.1016/0378-5173(96)04644-3

8. J. Siepmann, H. Kranz, and R. Bodmeier, Pharm. Res. 16, 1748 (1999). doi:10.1023/A:1018914301328

9. J. L. Ford, K. Mitchell, P. Rowe, D. J. Armstrong, P. N. C. Elliot, C. Rostron, and J. E. Hogan, Int. J. Pharm. 71, 95 (1991). doi:10.1016/0378-5173(91)90071-U

10. M. J. Vazquez, B. Perez-Marcos, J. L. Gomez-Amoza, R. Martinez-Pacheco, and C. Souto, A. Concheiro, Drug Dev. Ind. Pharm. 18, 1355 (1992). doi:10.3109/03639049209046332 
11. S. P. Vyas, N. K. Jain, and S. Khana, J. Control Rel. 10, 219 (1989). doi:10.1016/0168-3659(89)90065-5

12. D. M. Aviado and J. M. Porter, Pharmacotherapy 4 (6), 297 (1984).

13. M. Otsuka and Y. Matsuda, Journal of Pharmaceutical Sci. 83 (7), 956 (1994). doi:10.1002/jps.2600830708

14. P. Gao, P. Nixon, and J. Skoug, Pharm. Res. 12, 965 (1995). doi:10.1023/A:1016246028338

15. J. T. Carstensen, Pharmaceutics of Solids and Solid Dosage Forms (John Wiley and Sons, New York, 1977).

16. N. Shah, G. Zhang, V. Apelian, F. Zeng, M. H. Infeld, and A. W. Malick, Pharm. Res. 10, 1693 (1993). doi:10.1023/A:1018901509855

17. M. Refoio and In Encyclo, Polym. Sci. Tech. Suppl. 1: 214 (1976).

18. B-J. Lee, S-G. Ryu, and J-H. Cui, Drug Dev. Ind. Pharm. 25, 493 (1999). doi:10.1081/DDC-100102199

19. A, Kader and R. Jalil, Drug Dev. Ind. Pharm. 25, 141 (1999). doi:10.1081/DDC-100102154

20. U. S. Pharmacopeia (The United States Pharmacopeial Convention, Inc., Rockville, MD, 2004). 